\title{
El derecho al bienestar para la infancia indígena es una tarea pendiente de todos
}

\author{
The right to welfare for indigenous children is a task for all
}

En los últimos años se ha prestado creciente atención a todos los temas relacionados con los pueblos indígenas fundamentalmente en sus derechos y bienestar, constatándose progresos significativos en diferentes áreas y aunque alentador el proceso, los niños indígenas no han recibido aun la particular consideración que merecen.

En América Latina la Pobreza infantil indígena de acuerdo a datos de la CEPAL y UNICEF es de un $88 \%{ }^{(1)}$. En nuestro país los pocos datos existentes muestran un patrón de desigualdad más acuciante en la pobreza extrema, reflejadas por la privación severa en el acceso a la educación, al agua potable y a la vivienda, siendo tres veces mayor que la del resto de los niños. Esto expresa una violación de los derechos a la supervivencia y al desarrollo e implica altos costos para la sociedad en capacidades productivas e inclusión social. De allí la relevancia de analizar y monitorear la evolución de la pobreza para la formulación de políticas que permitan erradicarla.

En nuestro país se dispone de escasa información sobre los niños y niñas indígenas, respecto a sus condiciones de vida, su estado de salud, acceso a la escolarización, rendimiento escolar, protección contra el abandono, los abusos, la violencia y la explotación, lo cual explica la poca visibilidad de la dimensión de los problemas sociales que los afecta y por ende dificulta la promoción de mayor cantidad de estrategias y de asignación presupuestaria para ejecutarlas a favor de este grupo.

Los niños indígenas no tienen las mismas posibilidades de atención sanitaria que los no indígenas dado por el menor acceso a los servicios de salud. Esta situación se ve reflejada por tasas de mortalidad neonatal e infantil cifras significativamente mayores que las indicadas para la población nacional. La reducción de la mortalidad en menores de cinco años en nuestro país es fundamental, para lograr los compromisos establecidos en los Objetivos de Desarrollo del Milenio (ODM). Aunque no se tienen cifras muy claras, se estima que el perfil de mortalidad y morbilidad de los pueblos indígenas es similar al de los grupos socioeconómicos más desfavorecidos que se ven agravados por extrema pobreza, analfabetismo, desocupación y migración.

Es sabido que la infancia temprana es, junto con el estadio prenatal, el período más importante para garantizar el desarrollo saludable del niño, e iniciativas como los programas de nutrición e inmunización deben recibir un énfasis particular, la población infantil indígena presenta uno de los más altos niveles de pobreza y vulnerabilidad de todo el país, pues la prevalencia de desnutrición crónica de los niños indígenas casi duplica la de los niños no indígenas, y es más del triple que el promedio nacional, debemos interpretar la Salud como un derecho humano, una inversión social y no un gasto social.

El Paraguay cuenta con una población estimada al 2012 de 6.672 .631 habitantes $^{(2)}$, de los cuales 117.150 $(1.8 \%)$ corresponde a la población indígena, distribuidos en 19 pueblos pertenecientes a cinco grupos lingüísticos y habitan en 13 departamentos del país y en Asunción ${ }^{(3)}$. La elevada fecundidad es una característica asociada a la población indígena, sin embargo, en la última década ha disminuido de 6,3 hijos por mujer registrado en el censo anterior a 4,5 hijos en el censo 2012.

Los datos de la situación nutricional en niños 
indígenas considerando los criterios de las nuevas curvas de crecimiento OMS 2006 fueron obtenidos de la Encuesta Nacional a Hogares Indígenas EIH Indígena $2008^{(4)}$, encontrándose que el $41,7 \%$ presentaba desnutrición crónica, más del doble de la población infantil no indígena que acude a servicios del sistema público de salud correspondiente a ese mismo año $(18 \% \text {, SISVAN } 2008)^{(5)}$ la cual se asume corresponde a los quintiles más pobres. Así como también fue mayor al promedio nacional de 17,6\% para igual grupo etáreo no indígena según datos de la Encuesta Permanente de Hogares 2005 (EPH $2005)^{(6)}$.

En la situación nutricional de los niños y las niñas indígenas se distinguen diferentes niveles de causalidad: la situación de pobreza, la desigual distribución de la riqueza y el ingreso, siendo Paraguay uno de los países en América Latina con más inequidad en la distribución de ingresos, que afecta más a la población indígena, considerada como la mas excluida. Otras causas subyacentes son el bajo nivel educativo, la falta de acceso de agua potable y saneamiento básico, el acceso a servicios de salud y la falta de implementación de políticas públicas con una comprensión global y perspectiva intercultural del tema salud y nutrición. Abordar la atención en salud desde la interculturalidad es fundamental, entendiéndose la misma como la interacción comunicativa que se produce entre dos o más grupos humanos de diferente cultura, que pueden ser llamados etnias, sociedades, culturas o comunidades $^{(7)}$.

Cabe resaltar que se cuenta por primera vez con una Política de Salud Indígena y que el Programa Alimentario Nutricional Integral (PANI) sustentado por la Ley 4698/12 “De Garantía Nutricional en la Primera Infancia" brinda a los niños indígenas menores de 5 años de edad un modelo universal de atención independientemente de su estado nutricional a través de entrega gratuita de leche enriquecida y de la prestación de todas las ofertas sanitarias (controles de crecimiento y desarrollo, inmunización, charlas educativas, entre otras). En esta modalidad se incluye a toda la población indígena del Chaco y a algunas comunidades indígenas de la Región Oriental (Concepción, San Pedro Norte, Caaguazú, Itapúa, Alto Paraná, Central, Amambay y Canindeyú. A la fecha, el PANI asiste a 20.811 beneficiarios indígenas, de los cuales
17.376 son niños y niñas menores de 5 años y 3.435 son mujeres embarazadas.

Sin embargo, para garantizar su derecho básico a la alimentación, es necesaria la implementación de políticas públicas de seguridad alimentaria y nutricional para poblaciones indígenas, que visibilicen su situación y eleven su calidad de vida, puesto que, a pesar de la reducción de los niveles de pobreza y desnutrición en los últimos años, en ellos estos continúan siendo muy superiores al promedio nacional.

Al mismo tiempo, es fundamental la recuperación de las prácticas tradicionales de producción, preparación y consumo de alimentos, acompañada de la implementación de tecnologías para mejorar la disponibilidad de alimentos y la construcción de un modelo de gestión local que propicie el compromiso de los diferentes niveles de gobierno y de los actores locales públicos y privados en las comunidades, para responder con estrategias eficaces a sus necesidades específicas, y el desarrollo de capacidades en las familias indígenas para la producción diversificada de alimentos y el mejoramiento de sus prácticas de consumo, higiene y cuidado de la salud en base a un modelo de intervención intercultural, acorde a las condiciones y necesidades de la población indígena.

En este contexto, el INAN se encuentra coordinando actividades con el fin de contar con un diagnóstico sobre la situación alimentaria y nutricional de las poblaciones indígenas que servirá de línea de base para la elaboración de Guías Alimentarias para población indígena, como herramienta de educación alimentaria y nutricional con pertinencia cultural.

Son desafíos pendientes, superar la desigualdad, construir y lograr el abordaje con enfoque de interculturalidad, reconocer y valorar la diversidad, aumentar la cobertura de los servicios básicos, investigar y desarrollar líneas de base en relaciona a deficiencias de hierro, cobre y zinc, considerados micronutrientes esenciales entre otros.

Pretender un desarrollo social desatendiendo estas verdades insoslayables refleja la falta de congruencia entre lo que se expresa en el discurso y lo que demuestran las acciones concretas, sembrando dudas sobre el verdadero interés en 
modificar la realidad social y el futuro de las poblaciones involucradas. Por lo tanto el derecho al bienestar para la infancia indígena exige que trabajemos en cualquier nivel de atención, con compromiso humano pues es una responsabilidad de TODOS.
"Yo soy tu y tú eres yo, somos parte de un mismo tejido, somos parte de la tierra, Y ella es parte nuestra, somos parte del universo y somos parte de un todo" J. Simón

Laura Mendoza de Arbo Instituto Nacional de Alimentación y Nutrición Ministerio de Salud Pública y Bienestar Social

\section{REFERENCIA}

1. CEPAL (Comisión Económica para América Latina y el Caribe), UNICEF (Fondo de las Naciones Unidas para la Infancia). Guía para estimar la pobreza infantil: Información para avanzar en el ejercicio de los derechos de los niños, niñas y adolescentes. Santiago. CEPAL/ UNICEF; 2012.

2. STP (Secretaria Técnica de Planificación), DGEEC (Dirección General de Estadística, Encuestas y Censos. Paraguay: proyección de la población año 2012 según departamento. Asunción: STP/DGEEC;2012.

3. DGEEC (Dirección General de Estadística, Encuestas y Censos). III Censo Nacional de Población y Viviendas para Pueblos Indígenas 2012 y Censo Nacional de Población y Viviendas 2012. Asunción: DGEEC; 2012.
4. Sánchez S, Sanabria M, Medina N. Perfil nutricional de niños y niñas indígenas menores de 5 años de edad en el Paraguay. Encuesta a Hogares Indígenas 2008, DGEEC.

5. Instituto Nacional de Alimentación y Nutrición, Ministerio de Salud Pública y Bienestar Social. Sistema de Vigilancia Alimentaria Nutricional-SISVAN en Menores de 5 años, año 2008. Disponible en: www.inan.gov.py

6. Sanabria M. Análisis de la situación de salud infantil y antropometría en menores de 5 años, Paraguay EPH 2005. DGEEC/Naciones Unidas.

7. Comunicación Intercultural: conceptos y consecuencias. En: Austin Millán TR. Diálogos en la acción, primera etapa; 2004. 